\title{
Estudo da aplicação de uma abordagem de disseminação oportunística de dados no cenário de cidades inteligentes
}

\author{
Diego da S. Souza ${ }^{1}$, Pedro C. Silveira ${ }^{2}$, Jonice Oliveira ${ }^{1,2}$, Guilherme Fogaça ${ }^{1}$, Jano M. \\ de Souza ${ }^{3}$, \\ ${ }^{1}$ Programa de Pós-Graduação em Informática $-{ }^{2}$ Departamento de Ciência da Computação \\ ${ }^{3}$ COPPE - Programa de Engenharia de Sistemas e Computação - \\ Universidade Federal do Rio de Janeiro (UFRJ) - Rio de Janeiro - RJ - Brazil \\ \{diessouza, pedrosilveira, guilherme.fogaca\}@ufrj.br, jonice@dcc.ufrj.br, \\ janoecos.ufrj.br
}

\begin{abstract}
In order to prevent the information to be restricted to a single person or group, this paper presents an approach for the dissemination of various types of data in a mobile P2P network, through "bluetooth", promoting the exchange of knowledge opportunistically. To accomplish this, context information, as well as attributes of the user's profile are considered. We propose some scenarios of using this approach on Smart Cities. On this article we also show some analysis of the efficiency and extent of the spread of data in different scenarios. This approach, called MEK ${ }^{1}$, is being developed in partnership with Microsoft Research.
\end{abstract}

Resumo: Com o objetivo de evitar que informações fiquem restritas a uma única pessoa ou grupo, este trabalho visa apresentar uma abordagem de disseminação de diversos tipos de dados em uma rede P2P móvel, via "bluetooth", promovendo a troca de informações de modo oportunístico. Para realizar a troca de dados entre os usuários, informações de contexto, assim como características do perfil do usuário são consideradas. Propomos alguns cenários como utilização dessa abordagem para o ambiente de Cidades Inteligentes. Neste artigo também mostramos os resultados da análise da eficiência e do alcance da propagação dos dados em diferentes cenários. Esta abordagem, chamada MEK', está sendo desenvolvida em parceria com a Microsoft Research.

\section{Introdução}

Com o avanço de tecnologias "wireless", nossa forma de se comunicar com o mundo muda a cada dia. Os dispositivos móveis permitem que estejamos conectados permanentemente com as pessoas. Trabalhando com a hipótese de que o mau uso da informação ou até mesmo a falta dela é um problema que afeta os grandes centros urbanos, buscam-se novas alternativas que contornem ou melhorem esse fato. 
Este é o ponto central desta abordagem, chamada $\mathrm{MEK}^{1}$. As pessoas são consideradas como potenciais centros de conhecimentos ${ }^{2}$ onde quaisquer recursos de informação podem ser disseminados para quem o considere importante. A disseminação é realizada de maneira oportunística. Aproveitando as grandes inovações tecnológicas podemos pensar que cidades ou locais também podem ser consideradas fontes de dados. E a partir daí pode surgir uma colaboração entre os envolvidos - cidadãos, organizações e governo - de uma forma oportunística, de maneira que estes possam ser sorvedouros de informação para as pessoas que a consideram importante.

\section{Cidades Inteligentes}

De acordo com Giffinger et. al. [2007], há seis eixos a serem avaliados em uma CI: economia inteligente, mobilidade inteligente, ambiente inteligente, pessoas inteligentes, qualidade de vida inteligente e governança inteligente. Analisando estes itens, pode-se ter uma visão geral de como uma CI se comporta [Komninos 2006] e, a partir deles, traçar metas que melhorem o desempenho da cidade. Além disso, não se pode negligenciar o potencial que os dispositivos móveis têm de auxiliar na coleta de dados, captura de informações contextuais, gravação de relatos, fotos ou vídeos que permitem descrever ou registrar melhor um acontecimento.

Tendo em foco os dispositivos móveis e o conceito de CI, surge a possibilidade de uso do MEK. Fazendo o uso de rede P2P móvel, formada por um conjunto de objetos em movimento que se comunicam através de tecnologias sem fio de curto alcance, o MEK surge como uma aplicação que auxilia a troca de informações relevantes entre pessoas. Seu principal objetivo é capturar e transmitir dados do interesse do usuário, levando em consideração o local, o perfil do usuário (principalmente seus interesses), os conhecimentos que possui, os vizinhos existentes naquela localidade e instante de tempo, o perfil dos vizinhos, os conhecimentos que os vizinhos possuem, os temas do conhecimento. A partir disto é que a disseminação oportunística de conhecimentos para possíveis interessados é feita.

O MEK foi inicialmente avaliado para ser aplicado no cenário de healthcare, para que os envolvidos (profissionais da saúde, pacientes, familiares e amigos) possam colaborar, trocando dúvidas e conhecimentos sobre a doença, informações gerais, notícias e elementos de entretenimento que sejam de interesse em comum [Souza et. al. 2011]. Tendo os hospitais fornecendo informação útil às pessoas, colaboramos com três eixos das CIs: i)O pilar dos ambientes inteligentes, onde o hospital de uma forma oportunística pode fornecer informações relevantes para os seus pacientes, enfermeiros e médicos ou ainda para as pessoas próximas dos hospitalizados, podendo ainda analisar tais informações para o melhoramento contínuo do local; ii)o pilar das pessoas inteligentes, pois estas receberiam mais informações relevantes, aumentando o nível de conhecimento das pessoas que

\footnotetext{
${ }_{2}^{1}$ Mobile Exchange of Knowledge

${ }^{2}$ Nesta abordagem, todos os recursos trocados - dados em seus diferentes formatos - são chamados de 'conhecimento'
} 
estariam em contato com os hospitais; iii)por fim, o pilar de qualidade de vida inteligente, na qual pessoas teriam um maior acesso a informações, podendo colaborar para um aumento de sua qualidade de vida.

Embora o MEK seja avaliado no cenário de Saúde, seu uso é bem amplo, podendo atuar em outros cenários aplicáveis à CI, tais como: i)Identificação de demandas e ofertas Lojas podem colocar suas promoções e os interessados podem receber tal informação ao passar próximo da loja; ii)Troca de opiniões entre usuários - Opiniões sobre um local podem ser compartilhadas; Smart Hotel - Hóspedes podem receber em seus dispositivos informações sobre algum possível evento ou ofertas no hotel; iii)Utilidades Públicas - O governo poderia utilizar servidores implantados em obras públicas para fornecer informações sobre o andamento da mesma; iv)Trânsito -A medida que clientes de um estabelecimento se encontrem no estacionamento podem receber informações de engarrafamentos, vias obstruídas, acidentes, entre outros, para planejar melhor as suas rotas.

\section{Colaboração Oportunística}

De acordo com Kraut [Kraut et. al. 2006], existem 4 tipos de atividade interativa: planejadas, intencionais, oportunísticas e espontâneas. As interações planejadas são reuniões formais previamente marcadas. As interações intencionais são quando alguém procura explicitamente uma pessoa específica. As interações oportunísticas são interações que são antecipadas por alguém, mas só ocorrem se estas pessoas se encontram por acaso. As interações espontâneas não são esperadas por ninguém e ocorrem oportunisticamente. Segundo Zhang [Zhang et. al. 2006], o modelo de colaboração oportunística nos guia a um nível de colaboração mais responsável, pervasiva, flexível e distribuída, possuindo maiores vantagens para a difusão da informação e conhecimento.Esta forma de colaboração se assemelha a crowdsourcing [Howe 2006], o que pode ser muito benéfico para uma CI.

\section{Proposta - MEK}

O MEK possui diversas funcionalidades, tanto ativas, quanto passivas, para realizar a troca das informações. Todas as opções são acessadas pela interface principal da aplicação (Figura 1). Tais funcionalidades serão apresentadas a seguir.

\subsection{Perfil}

Tela onde ele poderá preencher informações tais como nome, idade e áreas de interesse. Os interesses são representados por conceitos em uma taxonomia já pré-definida. Um interesse pode conter diversas subáreas de interesse.

\subsection{Cadastrar Conhecimento}

Nesta etapa, o usuário pode cadastrar um conhecimento em seu aparelho e disponibilizá-lo para troca. O conhecimento é classificado pelo usuário através de assuntos definidos pela taxonomia de interesses. $\mathrm{O}$ conhecimento pode ter mais de uma área de interesse. 

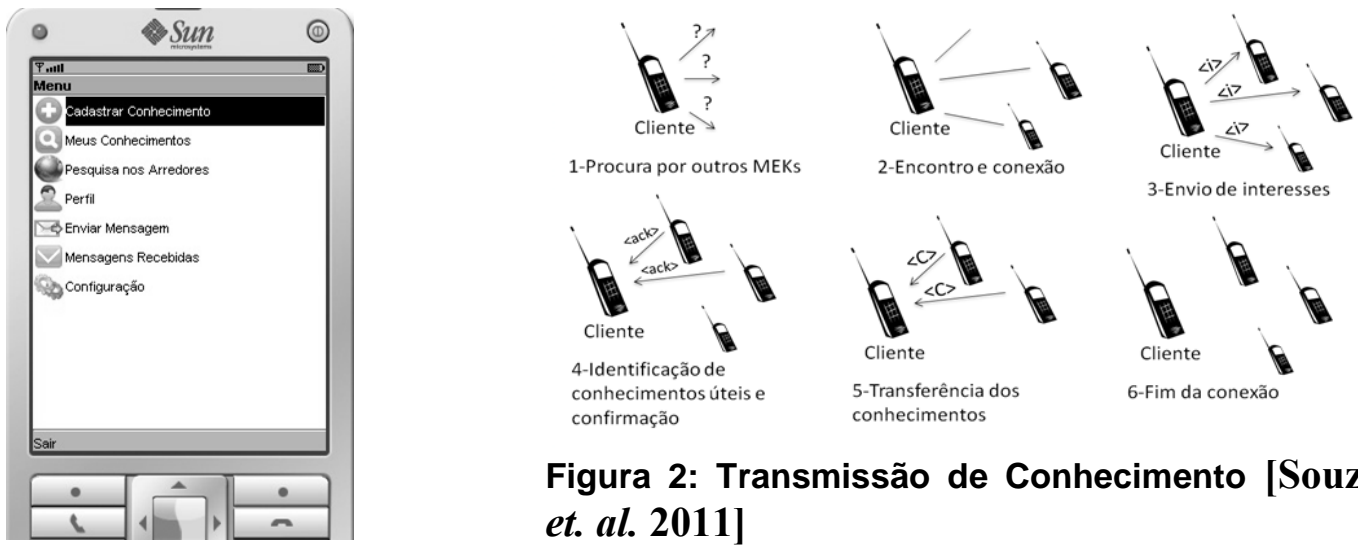

Figura 2: Transmissão de Conhecimento [Souza et. al. 2011]

Figura 1: Tela principal da aplicação MEK

\subsection{Troca de Conhecimento}

A troca de conhecimento é totalmente automatizada. O MEK, enquanto cliente, procura outros dispositivos próximos via interface bluetooth. Quando encontrado, eles conectam e a aplicação cliente envia o conjunto de interesses do usuário. A aplicação servidor verifica se possui conhecimentos que estejam classificados na mesma área ou sub-área de interesse. Caso possua, o MEK servidor sinaliza ao MEK cliente e este irá solicitar a transferência destes conhecimentos (Figura 2) [Souza et. al. 2011].

\subsection{Pesquisar}

O usuário pode buscar por conhecimentos já cadastrados em seu aparelho com o uso de palavras chaves, área de interesse ou título. Os conhecimentos podem ser agrupados por conhecimentos criados, conhecimentos adquiridos ou conhecimentos sugeridos.

\subsection{Pesquisar Arredores}

É possível para o usuário procurar ativamente por conhecimentos em aparelhos próximos usando novamente palavras chaves, área de interesse ou título. Uma vez selecionado um conhecimento, é iniciado sua transferência.

\subsection{Troca de Mensagens}

O MEK também disponibiliza a opção de troca de mensagens para um contato mais direto com as pessoas. Para uma maior privacidade para com os usuários o MEK disponibiliza uma opção de "Disponível para Chat", deste modo ele só é encontrado por outros aparelhos se esta opção estiver ativada.

\subsection{MEK Desktop}

Interface que permite o usuário organizar e sincronizar todo o seu conhecimento em seu computador, como: criação, edição e exclusão. 


\section{Avaliação}

Para analisar a eficiência da aplicação foram usadas as medidas de Precision (Equação 1) e Recall (Equação 2), a saber:

$$
\begin{gathered}
\text { precision }=\frac{\mid\{\text { conhecimentos relevantes }\} \cap\{\text { conhecimentos obtidos }\} \mid}{\mid\{\text { conhecimentos obtidos }\} \mid} \\
\text { Equação 1: Cálculo da taxa de precision }
\end{gathered}
$$

A análise da propagação dos conhecimentos foi realizada usando medidas como:

i. Número de conexões que falharam - Ou seja, os celulares não se 'acharam'.

ii. Número de conexões sem transmissão - Os celulares se 'acharam', mas não ocorreu a troca.

iii. Número de transmissões com sucesso - Os celulares se ‘acharam' e ocorreu a troca.

iv. Tempo de Transmissão (TT) - Tempo total - ou seja, duração de todas as trocas do cenário analisado.

Para a realização dos testes foi utilizada a taxonomia CNPq [CNPq 2011], para a classificação dos interesses.

Para a realização dos testes e execução dos cenários de testes foram utilizados 30 conhecimentos dos mais diferentes tipos de interesse. Duas versões de testes foram realizadas para cada cenário. Em um primeiro momento foram transmitidos conhecimentos sem anexos, e, por sua vez, em um segundo teste, foram utilizados anexos com tamanho variando entre $1 \mathrm{Mb}$ a $5 \mathrm{Mb}$. Estes anexos foram de vários formatos como música, som, vídeo, arquivos de textos, documentos do Word e PDFs.

Para uma melhor medição dos resultados foram utilizados 7 cenários de testes, com diferentes números de aparelhos, maneiras de transmissão e números de conhecimentos. Após a execução destes cenários, calculamos o número médio de conexões que falharam, de conexões sem transmissão e de transmissões com sucesso. Os resultados obtidos são apresentados em forma de tabela (Tabela 2). Calculando as taxas de Precision verificamos um aproveitamento de $100 \%$. O mesmo não ocorreu com a taxa de Recall, que apresentou um resultado verificado em aproximadamente $80 \%$. Em relação ao tempo total de transmissão, as trocas onde os conhecimentos não possuíam anexos (texto descrito diretamente no dispositivo), foram realizadas em curtos períodos de tempo (TT), não durando mais que 40 segundos. Já as trocas com anexos demoravam mais à medida que o tamanho do anexo crescia. Em nossos testes o tempo calculado (TT) variou de 40 segundos chegando até 2 minutos. 
Tabela 1: Resultados obtidos

\begin{tabular}{|l|c|c|c|c|c|}
\hline Números de Aparelhos & $\mathbf{2}$ & $\mathbf{3}$ & $\mathbf{4}$ & $\mathbf{5}$ & $\mathbf{6}$ \\
\hline Múdidas & 0 & 1 & 1 & 2 & 2 \\
\hline Número médio de conexões que falharam médio de conexões sem transmissão & 0 & 1 & 2 & 2 & 2 \\
\hline Número médio de transmissões com sucesso & 2 & 4 & 7 & 10 & 13 \\
\hline
\end{tabular}

\section{Agradecimentos}

À Microsoft pelo financiamento ao projeto "Temporal Social Networks and Knowledge Dissemination for Healthcares" (LACCIR, R1210LAC002), ao CNPq pelo apoio aos projetos "i-Presc: Identificação, Análise e Precisão de Redes Sociais Complexas" (processo: 476130/2009-3- Universal) e "LÁQUESIS: Mineração e Identificação do Fluxo da Informação em Redes Sociais Científicas" (processo: 308219/2010-4, PQ2), além de bolsas de estudos e CAPES por bolsas de estudos.

\section{Referências}

CNPq "Conselho Nacional de Desenvolvimento Científico e Tecnológico"(2011) http://www.cnpq.br/ , Acessado em Dezembro 2011

Giffinger R., Fertner C., Kramar H., Kalasek R., Pichler-Milanovic N., Meijers E. (2007) "Smart cities - Ranking of European medium-sized cities"

Howe J. (2006) "The Rise of Crowdsourcing" Wired Magazine - Issue 14.06- Vol. 14 - June 2006

Komninos N. (2006) "The Architecture of Intelligent Cities - Integrating human, collective and artificial intelligence to enhance knowledge and innovation" in 2nd IET

Kraut R. E., Fish R. S., Root R. W., Chalfonte B. L. (2002) "Informal Communication in Organizations: Form, Function, and Technology"

Souza D. S., Fogaça G., Silveira P. C., Oliveira J., Souza J. M. (2011) "MEK: Uma abordagem oportunística para disseminação colaborativa do conhecimento" in VIII SBSC

Zhang J., Scardamalia M., Revee R., Messina R. (2006) "Collective cognitive responsibility in knowledge building communities" 